\title{
PAPER
}

\section{A controlled study comparing visual function in patients treated with vigabatrin and tiagabine}

\author{
G L Krauss, M A Johnson, S Sheth, N R Miller
}

J Neurol Neurosurg Psychiatry 2003;74:339-343

See editorial commentary page 286 .

See end of article for authors' affiliations

.................

Correspondence to: Dr G L Krauss, Department of Neurology, The Johns Hopkins Hospital, Meyer 2-147, 600 North Wolfe Street, Baltimore, MD 21287-7247, USA gkrauss@jhmi.edu

Received 21 May 2002 Accepted in revised form 1 November 2002

\begin{abstract}
Objective: Vigabatrin treatment is frequently associated with irreversible retinal injury and produces retinal electrophysiological changes in nearly all patients. Concern has been raised that tiagabine and other antiepilepsy drugs (AEDs) that increase brain $\gamma$-aminobutyric acid (GABA) might produce similar electrophysiological and clinical changes in visual function. The study compared visual function between groups of patients with epilepsy treated long term with tiagabine, vigabatrin, and patients treated with other AEDs.

Methods: A cross sectional study comparing visual acuity, colour vision, static and kinetic perimetry, and electroretinograms between groups of patients treated with tiagabine, vigabatrin, and other AEDs (control patients). Patients were adults receiving stable AED treatment for $>6$ months.

Results: Vigabatrin treated patients had marked visual field constrictions in kinetic perimetry (mean radius $39.6^{\circ} \mathrm{OD}, 40.5^{\circ} \mathrm{OS}$ ), while tiagabine patients had normal findings (mean $61^{\circ} \mathrm{OD}, 62^{\circ} \mathrm{OS}$ ) (differences $\mathrm{OD}$ and $\mathrm{OS}, \mathrm{p}=0.001$ ), which were similar to epilepsy control patients (mean $60^{\circ} \mathrm{OD}$, $61^{\circ} \mathrm{OS}$ ). Vigabatrin patients had abnormal electroretinographic photopic B wave, oscillatory, and flicker responses, which correlated with visual field constrictions. These electroretinographic responses were normal for tiagabine patients and control patients. Patients were treated with vigabatrin for a median of 46 months compared with 29 months for tiagabine. Patients taking other AEDs that may change brain GABA had normal visual function.

Conclusion: Unlike vigabatrin, tiagabine treatment is associated with normal electroretinography and visual fields and ophthalmological function similar to epilepsy control patients. Differences between vigabatrin and other GABA modulating AEDs in retinal drug concentrations and other effects might explain why tiagabine increases in GABA reuptake do not cause retinal injury.
\end{abstract}

$\mathrm{V}$ igabatrin is associated with concentric visual field deficits in about $40 \%$ of treated patients. These defects appear to develop between three months and three years after the start of vigabatrin treatment and tend to persist despite cessation of the drug. ${ }^{1-3}$ The results of electrophysiological testing indicate that the major cause of the visual loss in patients taking vigabatrin is damage to the inner retina. ${ }^{1-5}$ Vigabatrin raises the concentration of $\gamma$-aminobutyric acid (GABA) in the brain by irreversibly blocking GABA transaminase, ${ }^{6}$ and it is believed that the retinal dysfunction that occurs in patients taking vigabatrin is related to a similar effect in the eye. Tiagabine blocks reuptake of glial and neuronal GABA. $^{78}$ The concern has therefore been raised that tiagabine and other antiepilepsy drugs (AEDs) that augment GABA neurotransmission might also produce visual deficits from their effects on the retina.

In a preliminary report, Beran et al reported that 6 of 12 patients treated with tiagabine developed visual field defects. ${ }^{9}$ More recently, Kaufman reported a similar case. ${ }^{10}$ Nousiainen, however, reported no visual field defects in 15 patients treated with tiagabine monotherapy. ${ }^{11}$ These conflicting findings may be attributable to methodological problems ${ }^{12}$; however, they raise the possibility that tiagabine might produce visual dysfunction. ${ }^{513}$

We performed a cross sectional, controlled study to determine if tiagabine causes electrophysiological and clinical changes in visual function similar to those associated with vigabatrin. We compared several measures of ophthalmological function-visual acuity, colour vision, funduscopic examination, visual fields using both static (Humphrey) and kinetic (Goldmann) perimetry, and electroretinography (ERG) - in patients treated with tiagabine, and in control patients with epilepsy taking other AEDs. These data were compared with patients treated with vigabatrin evaluated previously in an identical fashion.

\section{METHODS}

\section{Patients and inclusion criteria}

Patients were adult volunteers with epilepsy ( 12 tiagabine, 32 vigabatrin, and 14 control patients) recruited from the Johns Hopkins University epilepsy clinic and from other clinics in the US mid-Atlantic states (Maryland, Pennsylvania, New Jersey, Virgina, West Virginia, Delaware). Patients were excluded if they had pre-existing serious ocular disorders. Patients with previous exposure to vigabatrin were not included in the tiagabine and control study groups. Study consent was obtained for all participants using a consent approved by the Johns Hopkins University Joint Committee on Clinical Investigation.

Study patients had received treatment with tiagabine or vigabatrin for a minimum of six months at a stable dose and were permitted to take concomitant AEDs. The control patients had epilepsy and were group matched to tiagabine patients by age range, range of seizure frequency, and concomitant AEDs. Investigators performing the ophthalmological testing were blinded both to treatment conditions and to medical history. Control and tiagabine patients were tested in a randomised sequence. Vigabatrin patients were studied in a separate controlled study under identical conditions. Vigabatrin study results, but not vigabatrin and tiagabine comparisons, were previously published. ${ }^{14}$

Abbreviations: GABA, $\gamma$-aminobutyric acid; $A E D$, antiepilepsy drug; ERG, electroretinography; KVF, kinetic visual field 
Table 1 Clinical characteristics of patient population

\begin{tabular}{|c|c|c|c|}
\hline Characteristics & Vigabatrin patients & Tiagabine patients & Control patients \\
\hline Male/female & $20 / 12$ & $8 / 4$ & $7 / 7$ \\
\hline Age, mean years (range) & $43.1(22-73)$ & $42.2(26-70)$ & $40(21-71)$ \\
\hline Median duration of treatment, months (range) & $46.5(27-78)$ vigabatrin & $29(7-60)$ tiagabine & 0 \\
\hline Median monthly seizure frequency, (range) & $3.6(0-31)$ & $4.0(1-30)$ & $3(0-30)$ \\
\hline Median number of prior AEDs, (range) & $6(3-11)$ & $3(2-4)$ & $3(2-8)$ \\
\hline \multicolumn{4}{|l|}{ Number of patients taking concomitant AEDs } \\
\hline carbamazepine & 19 & 4 & 6 \\
\hline phenytoin & 9 & 3 & 7 \\
\hline gabapentin & 4 & 1 & 2 \\
\hline lamotrigine & 5 & 0 & 1 \\
\hline phenobarbittal & 0 & 1 & 4 \\
\hline primidone & 2 & 1 & 1 \\
\hline topiramate & 1 & 1 & 3 \\
\hline valproic acid & 4 & 0 & 3 \\
\hline levetiracetam & 0 & 2 & 3 \\
\hline \multicolumn{4}{|l|}{ Aetiology of seizures } \\
\hline idiopathic & 16 & 8 & 7 \\
\hline symptomatic & 16 & 4 & 7 \\
\hline
\end{tabular}

\section{Ophthalmology testing}

Every patient underwent a complete neuro-ophthalmological evaluation by a physician and a technician, both of whom were unaware of the patient's clinical status and medications. The evaluation included testing of visual acuity using a projected wall chart, pinhole visual acuity, colour vision using Hardy-Rand-Rittler (HRR) pseudoisochromatic plates, and visual field testing. Visual field testing was performed by kinetic perimetry using a Goldmann manual perimeter and by static perimetry using the 24-2 strategy on a Humphrey automated perimeter. During kinetic perimetry, fixation was carefully monitored by direct visualisation of the fixing eye, and catch trials (rechecking points both outside and within the area of apparently seeing field at specific meridia) were performed. During automated static perimetry, the foveal threshold was on, and reliability indices were automatically assessed. We subsequently excluded visual field results for one tiagabine patient with mental retardation whose responses during testing were unreliable during both kinetic and static perimetry; however, her ERG results are included in our analysis.

ERG b-wave amplitudes were measured as the difference in $\mu \mathrm{V}$ between the trough of the a-wave and the peak of the b-wave. ERG data were evaluated by comparison with a population of 100 normal subjects aged 20-80 years previously evaluated at Johns Hopkins and the University of Wisconsin, Madison. For all ERG signals, the best linear relation between age and parameter was determined and an equation was derived that described this relation. ${ }^{17}$ Individual subjects were considered to be abnormal if their data fell below two standard deviations of the age matched mean determined in this way. ERG amplitude and timing were also compared between vigabatrin treated patients and tiagabine and control patients using Student's $t$ tests.

Analysis of visual field data was performed by several methods. For kinetic perimetry, we used the V/4e test object and then determined the mean peripheral field diameter by measuring the radial distance of the intact field in degrees from fixation at each of 12 points, $30^{\circ}$ apart. We then averaged the results for each eye of each patient. We also averaged the results for the nasal and temporal homonymous hemifields separately. All results were compared with those obtained from the control patients. For static perimetry, we assessed the mean deviation for each eye and compared the results with those obtained from the control subjects. We also compared the performance of both patients and controls with respect to the reliability indices.

\section{RESULTS}

\section{Patient characteristics}

A total of 12 tiagabine patients, 32 vigabatrin patients, and 14 control patients were assessed. Age, sex, seizure frequency, and number of prior AEDs were similar among the treatment groups (table 1). Concomitant AEDs were similar except that none of the vigabatrin patients was taking levetiracetam, and none of the tiagabine patients was taking valproic acid or lamotrigine. The median duration of vigabatrin therapy was 46.5 months compared with 29 months for tiagabine treatment.

\section{Ophthalmological examination \\ Acuity}

There were no differences in acuity between tiagabine and control patients (OD, $\mathrm{p}=0.687 ; \mathrm{OS}, \mathrm{p}=0.202$ ). Three vigabatrin patients ( $9 \%$ ) had best corrected acuity of $20 / 40$ or worse OD, whereas no tiagabine patients or control patients had acuity worse than 20/20 OD. One tiagabine patient and one vigabatrin patient had 20/40 or worse acuity OS. However, there were no significant differences in acuity between tiagabine and vigabatrin patients $(O D, p=0.185$; OS, $\mathrm{p}=0.823)$.

Table 2 Results of visual field testing in tiagabine, vigabatrin, and control patients

\begin{tabular}{|c|c|c|c|c|c|}
\hline Technique & $\begin{array}{l}\text { Tiagabine patients } \\
n=12\end{array}$ & $\begin{array}{l}\text { Vigabatrin patients } \\
\mathrm{n}=32\end{array}$ & t Statistics & p Value* & $\begin{array}{l}\text { Control patients } \dagger \\
n=14\end{array}$ \\
\hline Kinetic OD & 61.5 & 39.6 & 4.5406 & 0.0001 & 60.1 \\
\hline Kinetic OS & 62.2 & 40.5 & 4.6117 & $<0.0001$ & 61.1 \\
\hline Static OD & $-1.98 \mathrm{~dB}$ & $-4.7 \mathrm{~dB}$ & 1.414 & 0.1647 & $-0.54 \mathrm{~dB}$ \\
\hline Static OS & $-1.67 \mathrm{~dB}$ & $-4.7 \mathrm{~dB}$ & 1.7266 & 0.0916 & $-0.54 \mathrm{~dB}$ \\
\hline
\end{tabular}




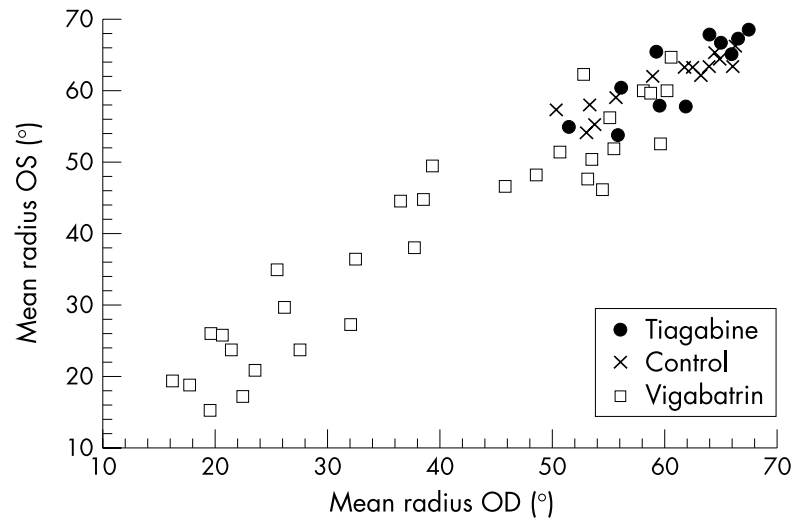

Figure 1 Kinetic visual fields: vigabatrin, tiagabine, and epilepsy control patients.

\section{Colour}

There were no differences in colour vision between tiagabine and control patients (OD, $\mathrm{p}=0.455$; OS, $\mathrm{p}=0.144$ ). One patient treated with tiagabine had slightly reduced (8/10) colour vision compared with normal values for control patients. Four vigabatrin patients $(9 \%)$ had abnormal colour vision $(<8 / 10)$.

\section{Visual fields}

Kinetic visual fields (Goldmann perimetry)

Vigabatrin patients had markedly constricted kinetic visual fields compared with tiagabine patients (vigabatrin treatment, mean radius $39.6^{\circ} \mathrm{OD}, 40.5^{\circ} \mathrm{OS}$; tiagabine patients, mean $61^{\circ} \mathrm{OD}, 62^{\circ} \mathrm{OS}$; both $\mathrm{p} \leqslant 0.001$ ), (table 2 and fig 1 ). Mean kinetic visual fields were nearly identical between tiagabine patients and control patients (mean $60^{\circ} \mathrm{OD}, 61^{\circ} \mathrm{OS}$ ) (table 2 and fig 1).

\section{Static visual fields (Humphrey perimeter)}

Vigabatrin patients had slightly greater static visual field mean deviations than tiagabine patients or control patients (vigabatrin $-4.7 \mathrm{~dB} \mathrm{OD},-4.7 \mathrm{~dB}$ OS); however, this did not reach statistical significance (table 2). Seven patients treated with vigabatrin, however, and no tiagabine or control patients had very high mean deviations $(>8)$. Mean static visual fields were normal for both tiagabine patients $(-1.9 \mathrm{~dB}$ OD,$-1.8 \mathrm{~dB}$ OS) and control patients ( $-0.54 \mathrm{~dB}$ OD, $-0.54 \mathrm{~dB}$ OS) (table 2$)$. One control patient and one tiagabine patient had slightly reduced kinetic and static visual fields on initial testing, but normal values on retesting. Overall, the controls and tiagabine patients had excellent reliability indices whereas the vigabatrin treated patients tended to have abnormal reliability indices that mirrored their visual field deficits.

\section{Fundoscopy}

All tiagabine patients had normal fundi as did all control patients. Among the vigabatrin patients, 23 (71.9\%) had abnormalities in the ocular fundi, including retinal arterial

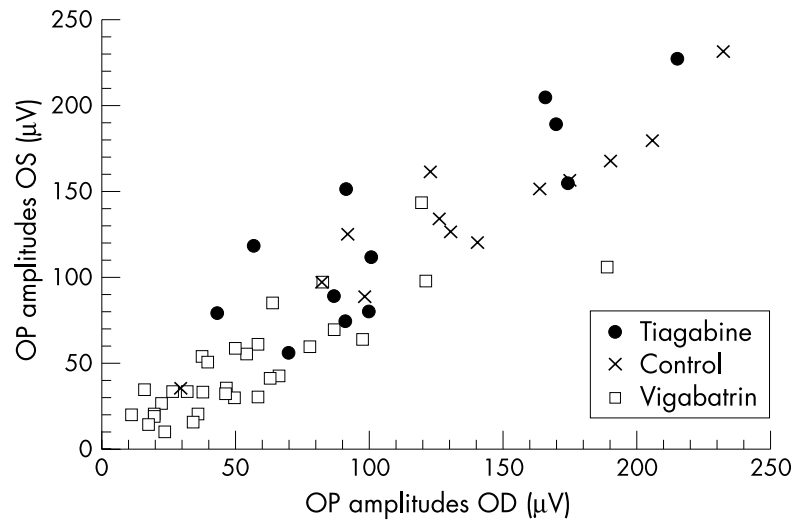

Figure 2 ERG oscillatory potentials: vigabatrin, tiagabine, and epilepsy control patients.

narrowing, epiretinal membrane formation, an irregular sheen or abnormal pigment in the macula, and reduction in the peripapillary nerve fibre layer. Two vigabatrin patients had pale optic discs.

\section{Electroretinogram findings \\ Cone system}

Vigabatrin patients had marked reductions in ERG photopic b-wave, flicker and oscillatory responses compared with patients treated with tiagabine, control patients, and with age matched laboratory norms (OD and OS, both $\mathrm{p}<0.001$ ) (table 3 and figs 2 and 3). Photopic b-wave, flicker responses and oscillatory responses were nearly identical for tiagabine patients and control patients (table 3 and fig 2 ).

\section{Rod system}

Vigabatrin patients had significantly diminished rod b-wave amplitudes (mean (SD) 103.1 (42) $\mu \mathrm{V}$ OD, 96.5 (41.7) $\mu \mathrm{V}$ OS) compared with tiagabine patients (140.92 (42) $\mu \mathrm{V}$ OD; 139.75 (44) $\mu \mathrm{V}$ OS; difference OD, $p=0.001$; OS $p=0.004)$, though the magnitude of these differences was smaller than for cone system driven responses. Rod b-wave amplitude for tiagabine patients were similar to control patients (151 (44) $\mu \mathrm{V}$ OD, $\mathrm{p}=0.6$; 151 (41) $\mu \mathrm{V}$ OS, $\mathrm{p}=0.5$ ).

\section{Visual field and ERG correlations}

KVF and photopic b wave amplitudes strongly correlated for vigabatrin treated patients, but not for tiagabine and control patients (fig 3). No significant correlations were shown between kinetic visual fields (KVF) and photopic b wave amplitudes, KVF and rod b-wave amplitudes, or SVF and photopic $b$ wave amplitudes for tiagabine patients.

\section{Other AEDs affecting GABA}

Eight patients in the epilepsy control and tiagabine groups were chronically treated with other AEDs that have been

\begin{tabular}{|c|c|c|c|c|c|}
\hline Technique & $\begin{array}{l}\text { Tiagabine } \\
\text { patients } n=12\end{array}$ & $\begin{array}{l}\text { Vigabatrin } \\
\text { patients } n=32\end{array}$ & t Statistics & p Value* & $\begin{array}{l}\text { Control } \\
\text { patients } n=14\end{array}$ \\
\hline Photopic OD & 89.5 & 48.4 & 5.3478 & $<0.001$ & 84.7 \\
\hline Photopic OS & 88 & 48.5 & 6.4162 & $<0.001$ & 88.8 \\
\hline Flicker OD & 68.6 & 31.9 & 6.3903 & $<0.001$ & 69.4 \\
\hline Flicker OS & 74.4 & 31.2 & 9.7805 & $<0.001$ & 72.1 \\
\hline Oscillatory OD & 113.8 & 55.5 & 4.014 & 0.0002 & 137.5 \\
\hline Oscillatory OS & 128.2 & 47.3 & 6.0772 & $<0.001$ & 136.8 \\
\hline
\end{tabular}




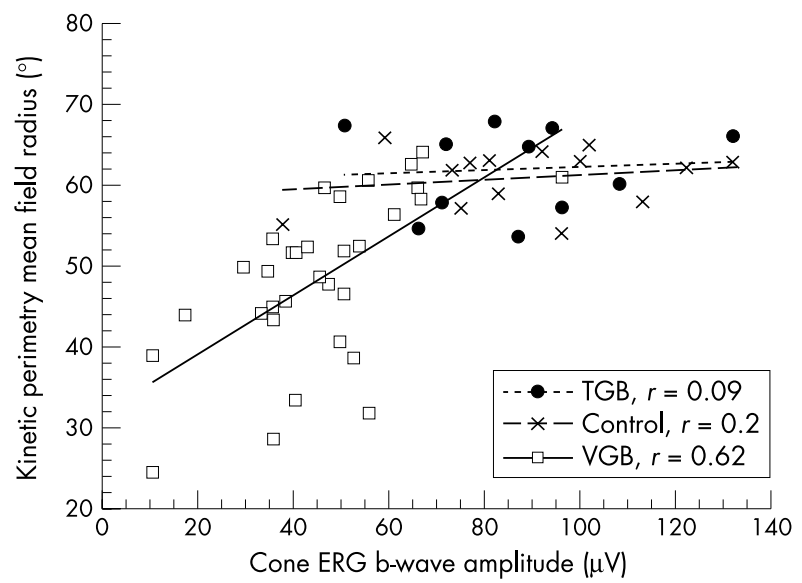

Figure 3 Kinetic visual fields versus ERG b wave amplitudes. Abnormalities in visual fields correlate with electrophysiological dysfunction in patients treated with vigabatrin. Results are normal for tiagabine and epilepsy control patients.

shown to modulate brain GABA: gabapentin, topiramate, valproic acid. These patients had normal visual findings, including normal KVF (mean 61.1 OD; 61.4 OS) and ERG flicker (mean 70.7 OD; 74.5 OS), testing that was abnormal in vigabatrin patients.

\section{DISCUSSION}

All vigabatrin treated patients had ERG abnormalities (decreased photopic b-wave, flicker or oscillatory potential amplitudes), and 53\% had visual field constrictions on KVF. Visual function testing for tiagabine treated patients, however, was similar to control patients with epilepsy. This is evidence that tiagabine is dissimilar to vigabatrin and does not produce clinically significant alteration in retinal function after intermediate periods of exposure (six months to five years).

The median exposure for patients treated with vigabatrin in our study was longer (46 months) than for tiagabine patients (29 months), however, vigabatrin patients treated for similar periods (one half of the total) had markedly abnormal ERG and visual fields similar to patients treated for longer periods. ${ }^{18}$ Only a small number of patients were treated with tiagabine for long periods (up to five years), however, our study shows there is no visual toxicity following intermediate periods ( $>6$ months) of exposure. We also found that KVF was more sensitive for detecting vigabatrin related peripheral field constrictions than SVF measured with 24-2 Humphrey perimetry. We did not, however, use the more sensitive 30-2 Humphrey protocol to measure static visual fields. Even though group differences in mean deviations for SVF did not reach statistical significance, many vigabatrin treated patients had large mean deviations in SVF, thus demonstrating a loss of visual sensitivity across central retinae in addition to their peripheral field constrictions demonstrated with KVF. Another limitation of our study was that vigabatrin patients were tested during a previous controlled study, however, test procedures were identical. Tiagabine and control patients were tested with investigators blinded to their treatment and no abnormalities were detected.

Vigabatrin has been associated with extensive pathological changes in all portions of the retina, including the ganglion cells and other cells in both the inner and the outer retina. ${ }^{1}$ We also have demonstrated widespread abnormalities in retinal electrophysiological function in patients treated with vigabatrin. ${ }^{19}$ Cone system function, particularly those responses dependent on inner retinal function-flicker and oscillatory responses-was most affected by vigabatrin. Abnormalities in flicker and oscillatory ERG responses depend on function of GABA rich cells in inner retina and correlated strongly with visual field deficits. This is evidence that vigabatrin retinal toxicity may be mediated by injury to GABA-ergic cells, particularly amacrine cells in inner retina that support cone function. This is consistent with our ERG and SVF evidence of loss of visual sensitivity across the retina, including central vision. As patients treated with tiagabine had normal cone system function demonstrated on ERG, with findings similar to epilepsy control patients and no correlation between ERG and visual field measurements, it is unlikely that tiagabine has physiological effects similar to vigabatrin in retina.

Our findings are consistent with studies showing different mechanisms of action and distributions for vigabatrin and tiagabine. Tiagabine reduces uptake of GABA in glia and neurons, whereas vigabatrin decreases activity of GABAtransaminase and blocks GABA metabolism in neurons and astrocytes. Unlike tiagabine, vigabatrin markedly increases GABA concentrations in cortical astrocyte cultures. ${ }^{2021}$ GABA levels in human brain are increased during vigabatrin treatment, whereas only small and variable changes are observed during tiagabine treatment. ${ }^{22}$ These differences in CNS effects extend to retina: vigabatrin concentrations in retina of rodents are increased fivefold compared with brain, while tiagabine concentrations are slightly decreased in retina compared with brain. ${ }^{23}$

Other AEDs shown to change brain GABA concentrations in addition to tiagabine-gabapentin, topiramate, and valproic acid-also did not change visual function. ${ }^{24}$ This is evidence against a drug class effect for GABA-ergic anticonvulsants producing retinal toxicity, though this does not exclude the possibility that vigabatrin produces retinal injury due to GABA alterations in retinas. Physiologically activated GABA receptors in isolated retina, for example, can produce increases in extracellular $\mathrm{Cl}^{-}$and rapidly trigger excitatoxic cell death. ${ }^{25}$

Patients with visual field constriction associated with vigabatrin tratment are often unaware of their deficits until and unless they become very severe. Such patients may have difficulties with navigation or visual adaptation, however, underscoring the importance of determining if drugs that affect the central nervous system produce retinal toxicity. Although our study is too small to determine whether rare patients develop visual complications during prolonged tiagabine treatment, our patients treated with tiagabine had retinal function similar to that of non-exposed patients with epilepsy. Moreover, vigabatrin caused retinal electrophysiological changes in all patients treated, with deficits in visual function occurring in a large proportion of exposed individuals. We found no evidence for similar disturbance in tiagabine patients, suggesting vigabatrin retinal toxicity is attributable to a comparatively specific effect on retinas.

\section{Authors' affiliations}

G L Krauss, S Sheth, N R Miller, Department of Neurology, Johns Hopkins University School of Medicine, Baltimore, USA

N R Miller, Department of Ophthalmology, Johns Hopkins University School of Medicine

M A Johnson, Department of Ophthalmology, University of Maryland School of Medicine

Competing interests: GLK and MAJ served as consultants to Cephalon Corporation one year ago, current manufacturer of tiagabine, and reviewed the study findings with them. The study was funded by Abbott Labs, former manufacturer.

\section{REFERENCES}

1 Krauss GL. Using the electroretinogram to detect and monitor the retinal toxicity of anticonvulsants. Neurology 2000;56:140-1.

2 Lawden MC, Eke T, Degg C, et al. Visual field defects associated with vigabatrin therapy. J Neurol Neurosurg Psychiatry 1999;67:716-22.

3 Johnson MA, Krauss GL, Miller NR, et al. Visual function loss from vigabatrin: effect of stopping the drug. Neurology 2000;55:40-5. 
4 Miller NR. Using the electroretinogram to detect and monitor the retinal toxicity of anticonvulsants. Neurology 2000;55:333-4.

5 Coupland SG, Zackon DH, Leonard BC, et al. Vigabatrin effect on inner retinal function. Ophthalmology 2001;108:1493-8.

6 Ben Menachem E. Vigabatrin. Epilepsia 1995:36 (suppl 2):S95-104.

7 Meldrum BS. Update on the mechanism of action of antiepileptic drugs. Epilepsia 1996;37 (suppl 6):S4-11.

8 Meldrum BS, Chapman AG. Basic mechanisms of Gabritil (tiagabine) and future potential developments. Epilepsia 1999;40 (suppl 9):S2-6.

9 Beran RG, Hung A, Plunkett $M$, et al. Predictability of visual field defects in patients exposed to GABAergic agents, vigabatrin or tiagabine. Neurology 1999;52 (suppl 2):A249.

10 Kaufman KR, Lepore FE, Keyser BJ. Visual fields and tiagabine: a quandary. Seizure 2001;10:525-9.

11 Nousiainen I, Mantyjarvi M, Kalviainen R. Visual function in patients treated with GABAergic anticonvulsant drug tiagabine. Clinical Drug Investigation 2000;20:393-400

12 Krauss GL, Miller NR. Vigabatrin: an effective antiepilepsy drug-balancing the risk of visual dysfunction. Ann Pharmacother 1999:33:1367-68

13 Stefan H, Bernatik J, Knorr J. Visual field due to antiepileptic drugs. [In German]. Nervenarzt 1999;70:552-5

14 Miller NR, Johnson MA, Paul SR, et al. Visual dysfunction in patients receiving vigabatrin: clinical and electrophysiologic findings. Neurology 1999:106:1792-8

15 Marmor MF, Arden GB, Nilsson SE, et al. Standard for clinical electroretinography. Arch Ophthalmol 1989;107:816-19.
16 Krauss GL, Johnson MA, Miller NR. Vigabatrin associated retinal cone system of dysfunction: electroretinogram and ophthalmologic findings. Neurology 1998;50:614-18.

17 Severns, ML, Johnson MA. Variability of the b-wave of the human ERG with intensity. Doc Ophthalmol 1993;85:291-9.

18 Paul SR, Krauss GL, Miller NR, et al. Visual function is stable in patients who continue long-term vigabatrin therapy: implications for clinical decision making. Epilepsia 2001:42:525-30.

19 Ravindran J, Blumbergs $\mathrm{P}$, Crompton J, et al. Visual loss associated with vigabatrin: pathological correlations. J Neurol Neurosurg Psychiatry vigabatrin: patho $2001 ; 70: 787-9$.

20 Sills GJ, Butler E, Thompson GG, et al. Vigabatrin and tiagabine are pharmologically different drug. A pre-clinical study. Seizure 1999;8:404-11.

21 Fraser $C M$, Sills GJ, Butler $E$, et al. Effects of valproate, vigabatrin and tiagabine on GABA uptake into human astrocytes cultured from foetal and adult brain tissues. Epileptic Disord 1999;1:153-7.

22 Petroff OA, Hyder F, Collins T, et al. Acute effects of vigabatrin on brain GABA and homocarnosine in patients with complex partial seizures. GABA and homocarnosine in

23 Sills GJ, Patsalos PN, Butler E, et al. Visual field constriction: accumulation of vigabatrin but not tiagabine in the retina. Neurology 2001;57:196-200.

24 Kuzniecky R, Ho S, Pan J, et al. Modulation of cerebral GABA by topiramate, lamotrigine, and gabapentin in healthy adults. Neurology 2002; 58:368-72.

25 Chen Q, Olney JW, Lukasiewics PD, et al. $\mathrm{Ca}^{2+}$-independent excitatoxity neurodegeneration in osolated retina, an intact neural net: a role for $\mathrm{Cl}$ and inhibitory transmitters. Mol Pharmacol 1998;53:564-72.

\section{NEUROLOGICAL STAMP}

\section{Allvar Gullstrand (1862-1930)}

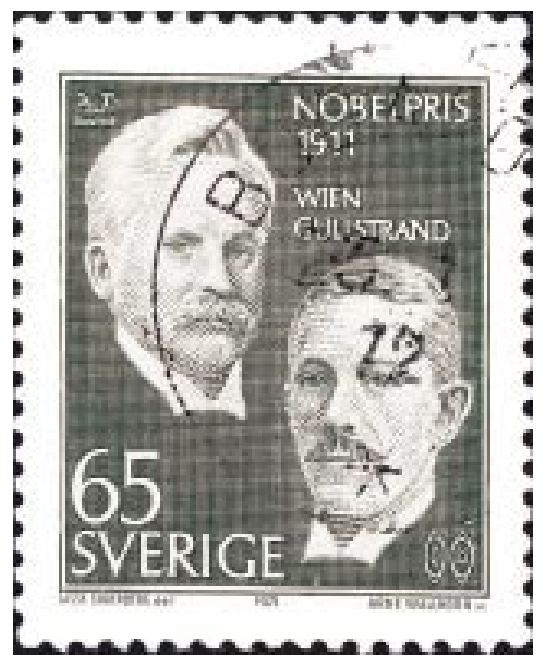

G ullstrand studied medicine at the University of Uppsala, graduated in 1887, and in 1894 became professor of ophthalmology. From his student days his career was devoted to the study of "dioptrics", that is, the study of the human eye as a transparent system of lenses for the collection and refraction of light. He invented a number of ophthalmological instruments. Among his contributions were the correction of astigmatism using cylindrical lenses, the localisation of paralysed ocular muscles, and in 1889 he invented a photographic technique for measuring the degree of corneal astigmatism. In 1911 Gullstrand expanded Hermann von Helmholtz's theory on the structure of the lens in accommodation. His most important technical achievement was in 1910, with the improvement of the ophthalmoscope, which had been invented by Helmholtz in 1851. This improvement was the precursor to the slit lamp Gullstrand invented in 1911. In that same year he received the Nobel prize for physiology and medicine. Gullstrand died in Uppsala in 1930. - Sweden honoured him philatelically in 1971 as a Nobel laureate (Stanley Gibbons no 671, Scott no 1910). He is shown with Wilhelm Wien, who received the Nobel prize for physics in the same year.

L F Haas 